\title{
THE ISCARSAH GUIDELINES ON THE ANALYSIS, CONSERVATION AND STRUCTURAL RESTORATION OF ARCHITECTURAL HERITAGE
}

\author{
PERE ROCA \\ On behalf of the ISCARSAH Subcommittee on the Guidelines \\ Universitat Politécnica de Catalunya (UPC-BarcelonaTech) \\ Jordi Girona 1-3, mod. C1, 08034 Barcelona, Spain \\ e-mail: pere.roca.fabregat@upc.edu,www.upc.edu
}

Keywords: Historical Structure, Architectural Heritage, Structural conservation and restoration

\begin{abstract}
The paper introduces the main concepts and criteria presented in the Guidelines on the Analysis, Conservation and Structural Restoration elaborated by the ICOMOS/ISCARSAH committee. The guidelines have been developed with the aim to provide guidance to experts and practitioners working in the study and conservation of structures or the architectural heritage. The guidelines are based on the ICOMOS Charter on the Principles for he Analysis, Conservation and Structural Restoration of Architectural Heritage elaborated by ISCARSAH and adopted by ICOMOS in 2003. The guidelines are intended to assist the multidisciplinary teams involved in the management of historical structures across all the stages of the study and operation, including the investigation and documentation works, the structural verification and the selection and design of appropriate minimum interventions.
\end{abstract}

\section{THE ICOMOS /ISCARSAH COMMITTEE AND THE GUIDELINES}

The International Scientific Committee on the Analysis and Restoration of Structures of Architectural Heritage (ISCARSAH) was founded by the International Council on Monuments and Sites (ICOMOS) in 1996 as a forum and scientific network of engineers, architects and scientists dealing with the structural dimension of monuments and historical constructions. The committee's focus is on the study and the conservation of the structures of cultural heritage buildings. The committee counts at present with more than one hundred international members.

The committee has been responsible for the elaboration of the ICOMOS Charter on the Principles for he Analysis, Conservation and Structural Restoration of Architectural Heritage [1] (ISCARSAH Principles) adopted by ICOMOS in October 2003. The ISCARSAH Principles, largely disseminated at the international level, have been translated to French, Italian, Spanish, Turkish, Arabic and other languages.

Jointly with the Principles, the ISCARSAH Committee has been working in a set of guidelines oriented to assist researcher and practitioners in the application of the Principles and, more generally, in the study of ancient structures across all its stages, including the investigation and documentation activities, the structural verification and the design of interventions. The 
ISCARSAH guidelines have been written with the aim to provide guidance to the multidisciplinary teams involved in the study and conservation of historical structures.

The approval in 2010 of Annex I on Heritage Structures of ISO13822 on Assessment of Existing Structures [2], elaborated with the contribution of ISCARSAH members, constituted an important step towards the dissemination of advanced concepts and practice on the study and restoration of historical structures. The ISCARSAH guidelines have been elaborated taking into account different relevant documents, including the Venice Charter of 1964 [3], the Nara Document of 1994 [4] and ISO13822 [2] along with the ISCARSAH Principles [1].

Following the ISCARSAH Principles and Annex I of ISO13822, the guidelines recognize that historical structures have cultural value in themselves and therefore their conservation must encompass the preservation of all tangible and intangible heritage values that contribute to their authenticity and cultural dimension. The structure is regarded as an essential part of the building's cultural legacy.

The guidelines are based on the premise that a combination of both scientific and cultural insight and knowledge is indispensable for the study and care of the architectural heritage. The necessary insight and knowledge are provided by the different experts integrated in the multidisciplinary team. The guidelines are also based on the understanding that, within such a multidisciplinary approach, structural engineering can provide the technical support necessary to safeguard the structure not only as a functional device but also as a cultural asset.
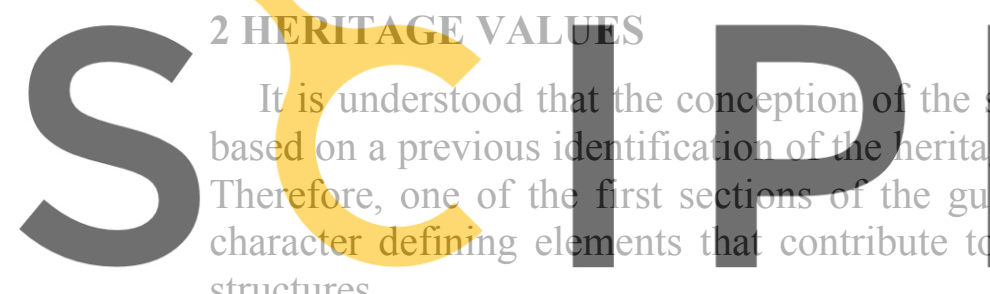
character
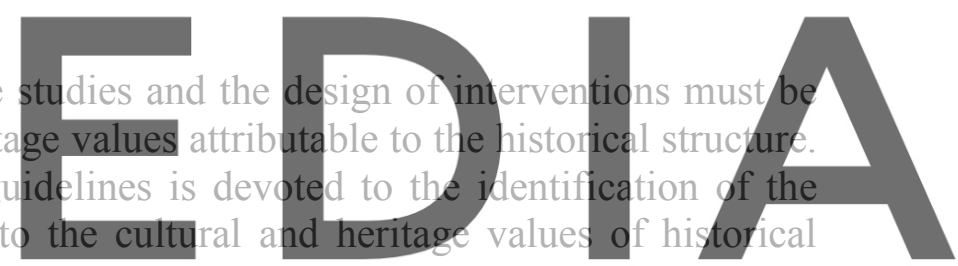

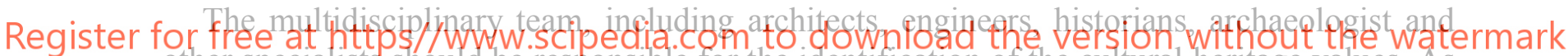
other specialists should be responsible for the identification of the cultural heritage values. As for any cultural resource, the authenticity and the heritage values of a historical structure encompass both tangible and intangible values. Among the tangible character-defining elements, providing heritage value, are the geometry, the materials, the overall structural organization, the construction details and connections, the location and setting (the environment). Imperfections and alterations may be also identified as character-defining elements, and should be respected provided that they do not compromise the safety requirements or the structure's durability. Among the intangible character-defining elements are the construction traditions and techniques, the structural concepts, the original (and subsequent) use and function, and the sources of evidence on ancient knowledge and technology. The spirit and feelings conveyed by the historical construction are also regarded as an intangible character-defining element.

The identification of the character-defining elements and the assessment of the heritage values are of upmost importance for the design of minimum interventions. The evaluation of the impact of the interventions on heritage values is a critical operation in the application of the scientific and engineering approaches presented in the following sections. 


\section{MINIMUM INTERVENTION}

A key concept referred to in the guidelines is that of the minimum intervention. A minimum intervention is the defined as an intervention that optimally combines compliance with structural requirements with the maximum possible protection and enhancement of heritage values and respect to the structure's authenticity. The guidelines provide significant attention to the concepts and procedures concerning investigation and operation which may lead to an adequate minimum intervention. In any case, the definition of minimum intervention involves the need to assess the impact that any intervention will have on the heritage values, which in turn requires to provide criteria on how such evaluation may be performed.

In addition to preserving the structure's authenticity and heritage values, minimum interventions are also desirable for other meaningful reasons. On one hand, excessive unnecessary alteration (incurred by a non-minimum intervention) is not advisable because the modified structure may develop a response difficult to predict, especially in the case of earthquake, with potential undesirable consequences. Furthermore, solutions causing a significant alteration may be very difficult or impossible to remove, therefore compromising later improvements or future new interventions, as dictated by the principles of removability or re-treatability later discussed.

In the guidelines, and based on large previous experience by ISCARSAH members, structural performance and conservation are regarded as complementary conditions leading to convergent optimum operations through the concept of minimum intervention. Rather than being in contradiction, it is understood that,
structural point of view are also the ones b
structure.
4 PROPOSED APPROACH
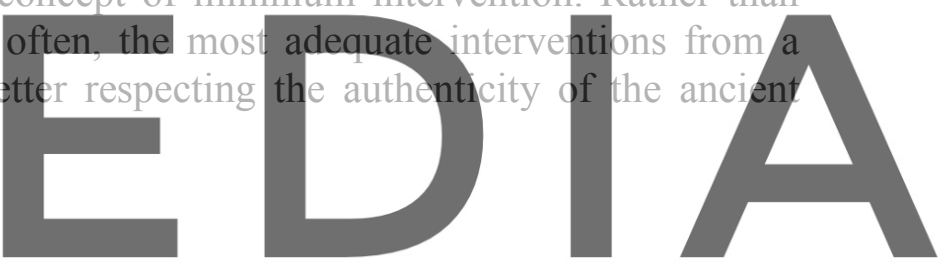

It is understood that an acritical application of codes and standards not specifically oriented

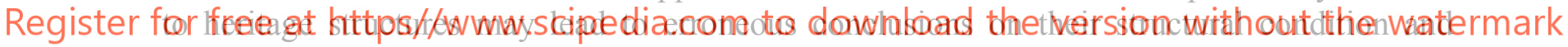
intervention needs. In specific, the rigid application of seismic and geotechnical codes may lead to misinterpret the resistant nature and real capacity of the historical structure and to apply drastic and unnecessary strengthening measures. Similarly, conventional methods for structural analysis oriented to new construction are often based on hypothesis on the structural behaviour that may not be applicable to historical structures.

While the overdesign caused by conservative approaches may be acceptable for new construction, a too conservative approach to structural safety may not be acceptable in the case of heritage structures due to excessive and unjustifiable cost in terms of loss of authenticity and cultural value. Actions that are not indispensable for the safeguarding of the building should be avoided.

In an attempt to overcome the aforementioned difficulties, a different approach is proposed strongly based on the scientific method. The approach proposed integrates qualitative and quantitative evidence through the combination of a set of complementary activities. These activities are:

- Historical research, based on the study of the relevant historical facts on the structure 
through the investigation of all kind of documentary sources.

- Inspection consisting in the characterization of the current condition and properties of the materials and structure through surveys and investigations.

- Monitoring, based on the observation of the response of the structure over a period of time.

- Structural analysis, consisting in the quantification of the response of the structure by means of a sufficiently accurate and calibrated structural model. Calibration and validation of the model is done by comparison with empirical information provided by historical investigation, inspection and monitoring according to the approach later described.

These activities are consistently developed across the different phases of the study, which include

(1) the assessment and investigation works, oriented to identify the causes of damage (diagnosis) and to conclude on the structure's conservation condition,

(2) the structural verification, involving the characterization of the structural performance and the identification of the intervention needs, and

(3) the design of the intervention, including the identification and full definition of optimal remedial solutions.

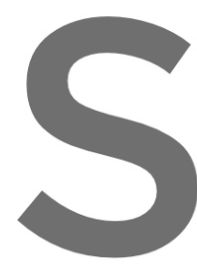

5 ASSESMENT. INVESTIGATIONAND DOCUMENTATION WVRIKS
The first phase of the study includes all the necessary investigations to co
current condition and causes of decay and damage. For this purpose, it is imp
investigating team incorporates the wide range of skills that may be necessary for

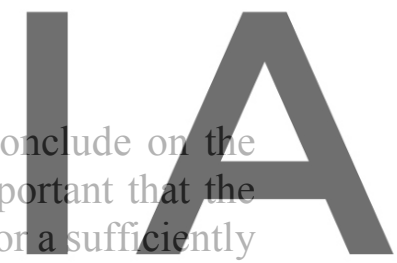

deep understanding of the structure and its problems. The process leading to the necessary

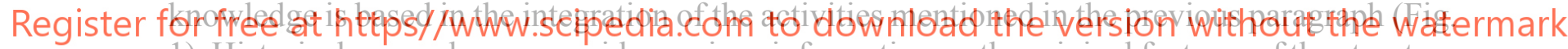

1). Historical research may provide precious information on the original features of the structure

and latter historical facts or events having had a sensible impact on it. Inspection involves all

the necessary activities to characterize the current properties and condition of the structure.

Monitoring may provide quantitative information on the evolution of the response of the structure along the monitored period. Finally, structural analysis can contribute to the characterization of the response of the structure for a variety of conditions and actions.

Historical research is regarded as an essential activity that may provide valuable information on the original features of the structure and latter historical events having had impact on it. Aspects to investigate are the stricture's historical and cultural significance, the original construction materials and procedures, the subsequent changes affecting both the structure and its environment, historical natural actions (such as earthquakes) or anthropogenic ones, and past restoration works.

The inspection works can be divided into two different stages, corresponding to (1) the survey of the structure by direct observation and measurements and (2) in situ and laboratory testing. The main objectives of inspection by direct observation include the characterization of the construction and structural features, the identification of ongoing environmental effects and the identification and mapping of damage. The preliminary inspection of the building allows 
also the location of adequate spots for the performance of geophysical surveys, the execution of Non-Destructive Tests (NDTs) and Minor Destructive Tests (MDTs) and the extraction of samples to be tested in laboratory. As part of the direct observation stage, decisions should be taken on immediate risks and the need for urgent stabilization measures. The second inspection stage (in situ and laboratory testing) involves a detailed characterization of the materials and structure, including their deterioration processes.
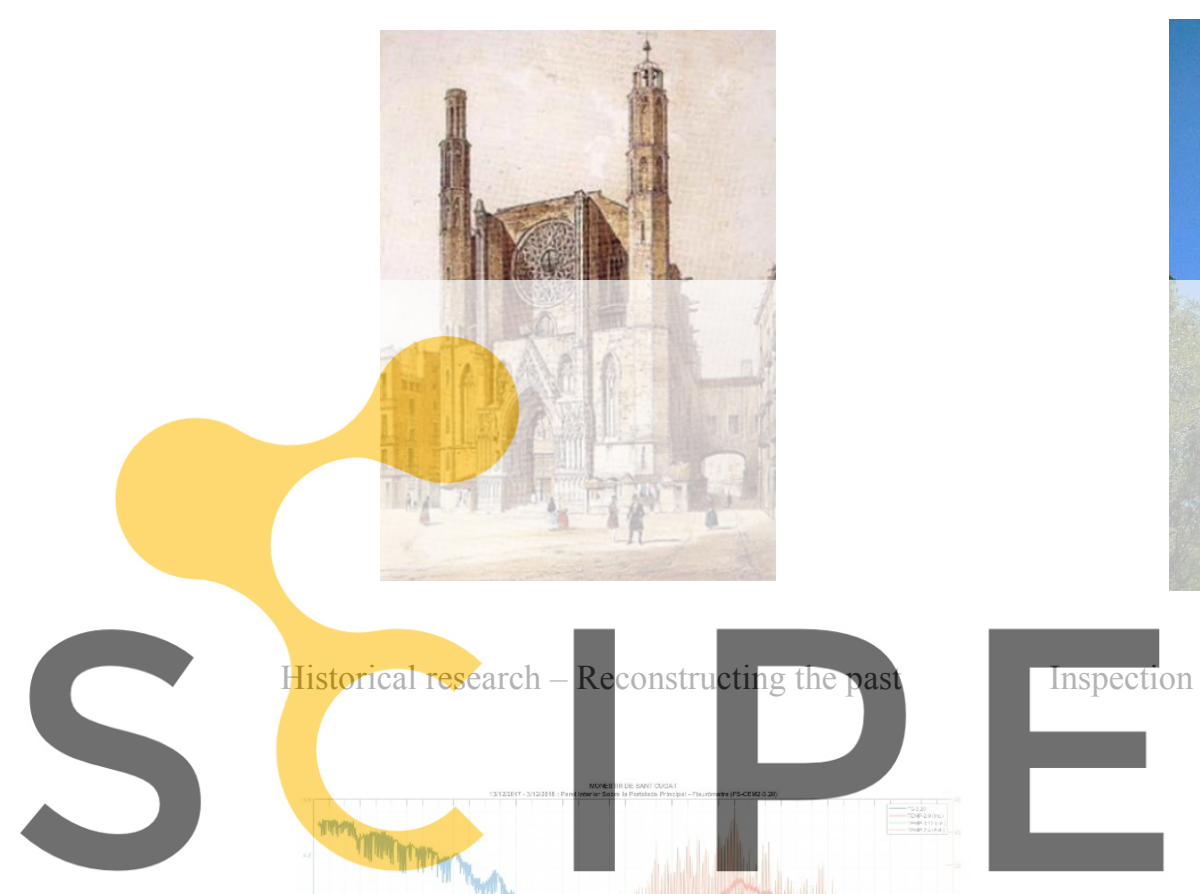

Register for free at $h$ ttps//www.scipedia.com/to

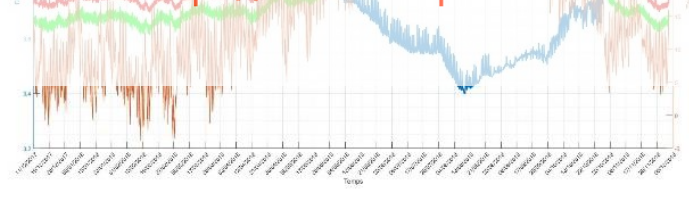

Inspection - Identifying the current trends
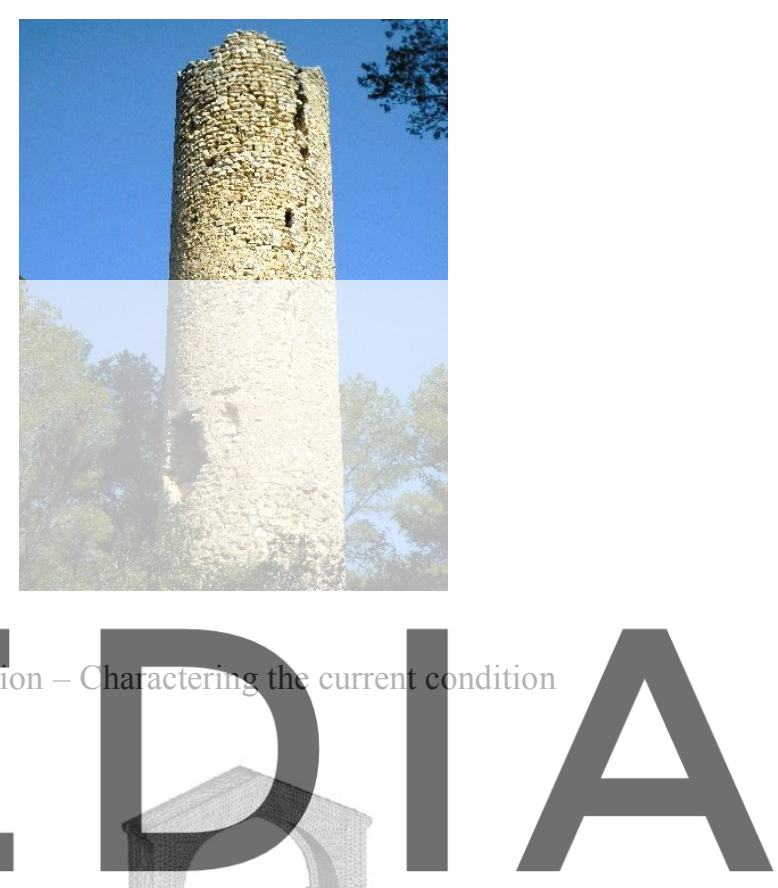

download the version without the watermark

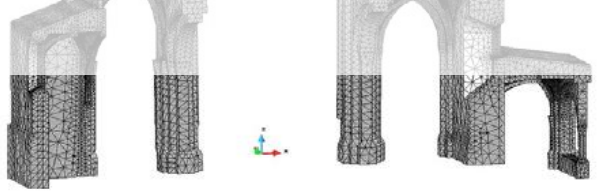

Structural analysis - Simulating past actions

Figure 1: The activities than can be integrated during the investigation and diagnosis phase to draw conclusions on the causes having induced damage and the present structural condition

The guidelines distinguish between static monitoring, consisting in the recording of structural parameters (such as displacements, rotations, crack openings, stresses, etc. ...) over a long period of time, and dynamic monitoring, used to record the response of the structure during the occurrence of seismic events, strong wind episodes or other episodic (normally dynamic) actions. Some criteria are provided on how to apply both types of monitoring. In specific, regarding static monitoring, some requirements are mentioned as necessary for an adequate post-processing and interpretation of the registered data. These requirements are (1) 
the identification of the critical variables (movements, crack widths), (2) the measurement of not only structural parameters (as crack openings) but also environmental ones (wind speed, temperature and humidity) and (3) a sufficiently long monitored period. Monitoring may be oriented to either survey the evolution of a non-intervened structure or to assess the effectiveness of a possible intervention.

During the diagnosis phase, structural analysis can be utilized to investigate the performance of the structure under past actions. These actions may include gravity loads, earthquake, wind, soil settlements, chemical or physical attack, anthropogenic alterations and thermal cycles, among other. Structural analysis can be utilized to characterize the possible contribution of different actions to existing damage and possible partial collapses. Structural analysis always involves the adoption of a model whose hypotheses and assumptions should be carefully chosen and taken into consideration in the interpretation of the results.

\section{ASSESSMENT. STRUCTURAL PERFORMANCE VERIFICATION}

The guidelines recognize that the structural verification of a historical structure constitutes a genuine problem posing significant challenges to the analyst. In any case, it is a problem fully encased within engineering understanding and equally solvable by means of engineering approaches, even if the contribution of the multidisciplinary team is of upmost importance. According to the guidelines, the verification of heritage structures involves a multi-dimensional problem in which the conventional and ultimate limit states are considered along with the

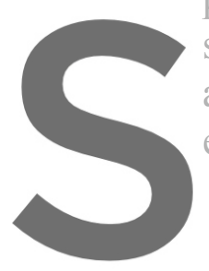
safeguarding of the possible authenticity and cultural value. evaluated taking into

(1) The safety of people at risk
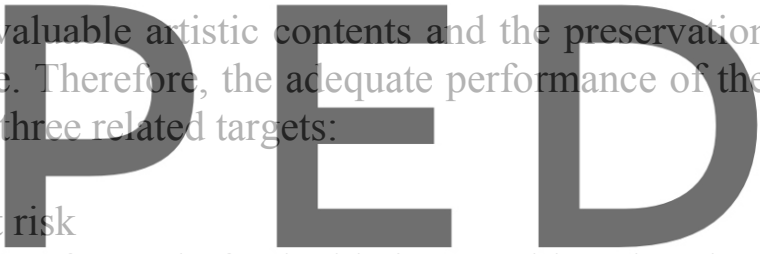

(2) The maximum possible safeguard of valuable immovable cultural and artistic contents

\section{Register for fire The inttegrity/ofthe striftudi as a chl turad herifage gbiect version without the watermark}

Objectives (1) to (3) will lead to the definition of the acceptable damage levels and the target reliability levels to be considered in the structural verification.

In the presence of very valuable paintings or frescoes fixed to walls or vaults, or other immovable cultural contents, and as suggested by the Annex I of ISO13822, condition (2) may be implemented through the consideration of specific serviceability limit states oriented to control deformation and damage.

In some cases, and as also indicated by Annex I of ISO13822, the protection of the structure's cultural value may lead to the acceptance of reliability levels lower than those implicit in building design codes. In these cases, parallel measures such as restricted use or provision of alternate escape routes must be adopted to limit the consequences of a failure on people. The method presented in section 7.3 provides a certain understanding on the situations that may require the reconsideration of the acceptable reliability levels.

The structural performance verification is also based on the general approach described in section 4 and integrates similar activities. Four different approaches can be considered (Fig.2):

- Historical approach, based on the investigation of the past performance of the structure. 
- Qualitative (or comparative) approach, based on the comparison of the structure being analysed with a number of similar constructions whose behaviour is already understood.

- Experimental approach, consisting in the execution of on-site experiments aimed to measure the load capacity of structural members.

- Analytical approach, based on the use of structural analysis to predict the performance of the structure under future actions.

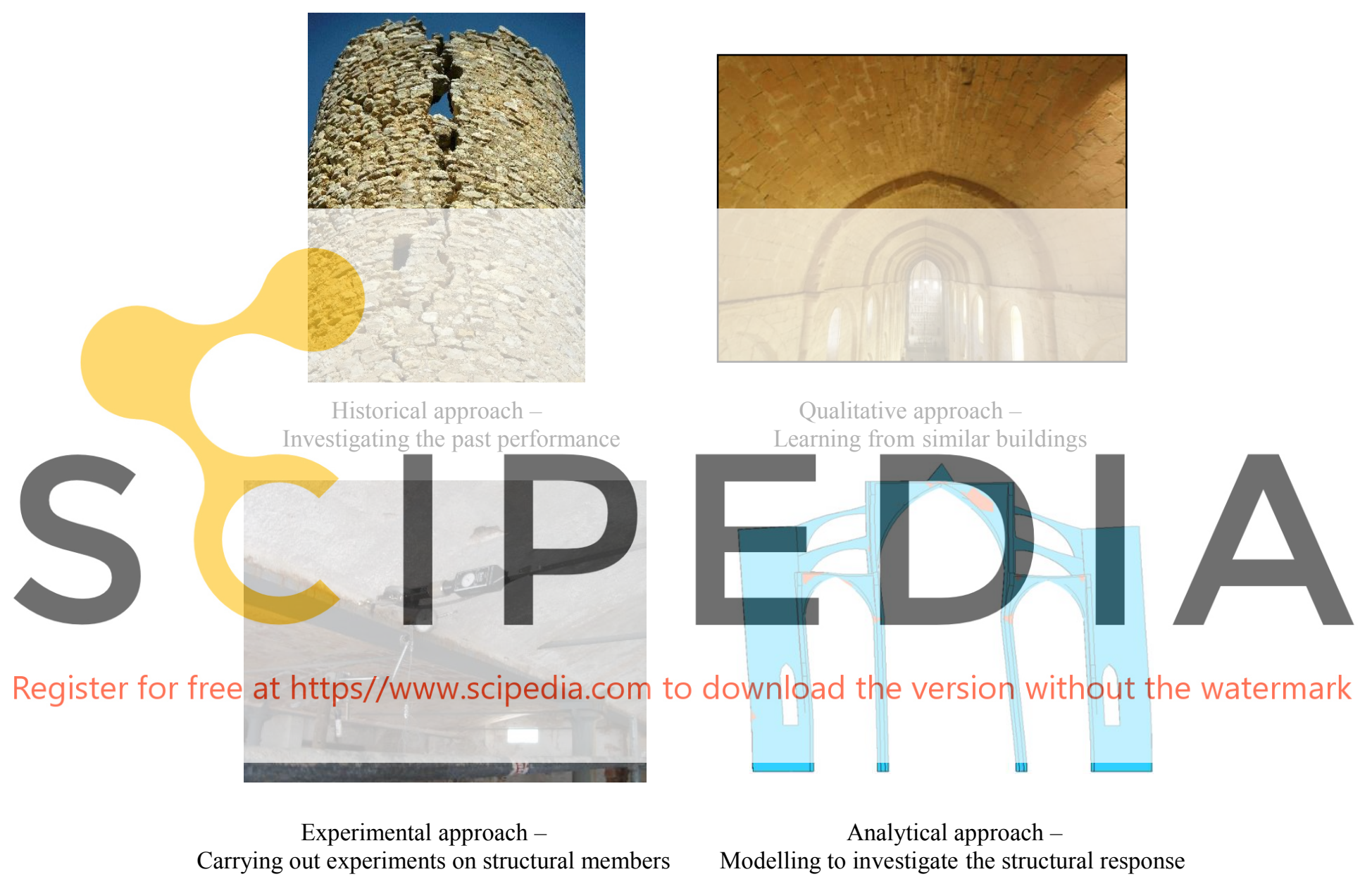

Figure 2: Different approaches that can be activated and integrated to conclude on structural performance

In the context of the structural performance verification, structural analysis is regarded as a deductive procedure in which the information on the structure, morphology and actions is invested (as input data) in constructing a structural model which is then used to predict the structural performance under future actions.

It is recognized, however, that structural analysis may suffer from significant uncertainty due to the fact that their reliability depends largely on the representativeness and realism of the adopted mathematical formulations. It is also recognized that data are always limited and liable to uncertainty. Often, the amount of data available are largely insufficient for a complete definition of a model. Due to these uncertainties, models must be always calibrated using the 
available evidence on the performance and condition of the structure. This evidence may be provided by historical analysis, inspection and monitoring. However, a full validation is not actually possible and significant uncertainty may remain in spite of the calibration effort. Engineering judgment is therefore necessary to evaluate the feasibility and reliability of the predictions and the correct use of the entire procedure leading to model construction, calibration and prognosis.

The guidelines devote a full section to the approaches and technical possibilities for model calibration. Among other possibilities, this section refers to calibration by comparison of the numerical predictions with existing crack patterns, historical past performance, stress states as measured by means of flat-jack tests (or other procedures), dynamic response or static monitoring response.

The activation of the above four approaches also requires for a methodology or criteria for the integration of their possible outcome. Several criteria are provided on how to combine and integrate the approaches. Such criteria consist on the principles of consistency, completeness and corroboration. Consistency refers to the need for identifying possible contradictions among the information provided by the approaches (the "plausibility check"), in which case improved investigations are needed until the contradictions are explained and solved. Completeness concerns to the need to obtain the necessary and sufficient information on the relevant variables of the problem. Corroboration refers to the convenience or need for redundant evidence provided by the different approaches as a way to confirm the information inferred. When the evidence obtained through the different appro
additional effort may be needed to improye the
In any case, the process leading to the verdi
synthesis of the approaches, is not formatized
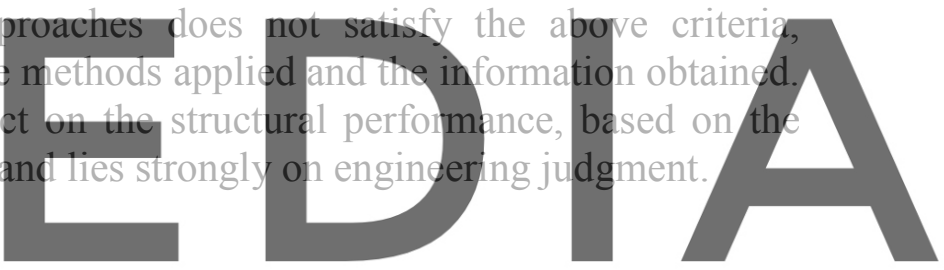

\section{Register foll finerathtepstitwwin.scipedia.com to download the version without the watermark}

A set of criteria or principles oriented to better identify and develop adequate minimum interventions are provided and discussed. Some of these criteria are in fact well known and are currently adopted in studies or projects on interventions in historical structures. Among them are the principles of compatibility with the original material and techniques (including chemical, physical, mechanical, rheological and thermal compatibilities), durability of the repair or reinforcing materials, non-invasiveness and removability of the devices or technologies used for strengthening.

According to the removability criterion, it must be possible to remove and replace the adopted measures. Replacement of the measures may be necessary when the former ones do not prove sufficiently efficient or to implement a more technologically advanced and respectful solution. Removability implies that the dismantlement of the intervention will only generate limited and repairable deterioration on the original construction.

A new criterion, designed as re-treatability, is introduced in the guidelines. According to it, any intervention should be designed in such a way that it does not compromise future interventions. This principle is of upmost importance when the technologies utilized do not comply with the aforementioned principles, and especially with non-invasiveness and 
removability.

According to the criterion of controllability, it must be possible to control the intervention during its execution. Measures that are impossible to control should not be allowed.

Last but not least, the guidelines refer to the principle of sustainability, according to which restoration projects should be mostly based on sustainable materials and technologies with low environmental impact during their life cycle.

It is recognized that applying all the mentioned criteria in a simultaneous and strict way may be difficult or impossible in practice. Instead, the criteria should be regarded as convenient guidelines or inspirational principles to be applied to the extent possible in the design of solutions approaching the concept of minimal intervention. For instance, lime mortar grouting for the consolidation of masonry walls may be regarded as an acceptable (even very satisfactory) solution in spite of its invasive character due to its proven durability and compatibility. In turn, strengthening solutions using a material with limited durability (as steel) may be acceptable if they are applied in a non-invasive and removable way and can be regularly inspected and maintained.

\subsection{Pro and post natural hazards structural maintainability}

The concept of structural maintainability is a novel contribution of the guidelines. The inspiration of the concept lies on real practices undertaken traditionally in seismic regions. In sùch locations, historical structures have been subjected to repeated repair or strengthening operations during their history. Rather than introducing drastid transformations, the traditiond
practice has mostly involved regular operations consisting in repair and maintenance. In a
similar way, it is understood that the modern approach to seismic resistance of historical
structures should base on an extended eoncept of maintenance allowing the preservation, as far
as possible, of the original construction characteristics. Structural maintanability is defined as a continuous maintenance programme involving minimal repair or strengthening operations undertaken after each earthquake. Such interventions should be designed so that they do not

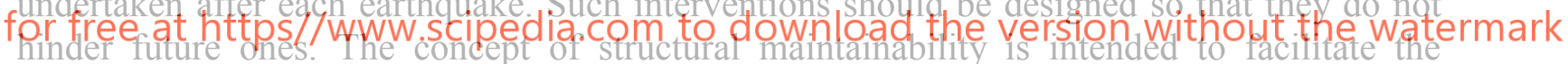
application of the principle of minimum interventions to the case of buildings affected by earthquake and other natural hazards.

\subsection{Proposed engineering approach to the design of the intervention}

The proposed approach for the selection and design of interventions is summarized in the flowchart of Fig. 3. The approach is based on the common engineering procedure consisting of envisaging and evaluating a set of alternative solutions. It is also based on the evaluation of the impact of the different alternatives on the heritage values.

After the definition of the reliability levels, a number of alternative solutions may be envisaged satisfying as far as possible the criteria presented in section 7.1. All these solutions should attain the targeted structural reliability levels. In addition, all solutions must be evaluated regarding their impact on the heritage values. Among these solutions, the one causing the minimum impact to heritage values should be selected. If the impact of this solution is sufficiently small (in the sense that its benefit largely surpasses its impact on heritage values), then this solution can be considered as a satisfactory minimum intervention. Otherwise, if the impact on the heritage values is deemed excessive, the adopted structural requirements may be 
reconsidered and possibility reduced (which, as mentioned, would require the adoption of complementary measures to avoid inacceptable risk on people). Adopting modified reliability levels means to reinitiate the process and again envisaging possible alternative solutions from which to derive a possible minimum intervention.
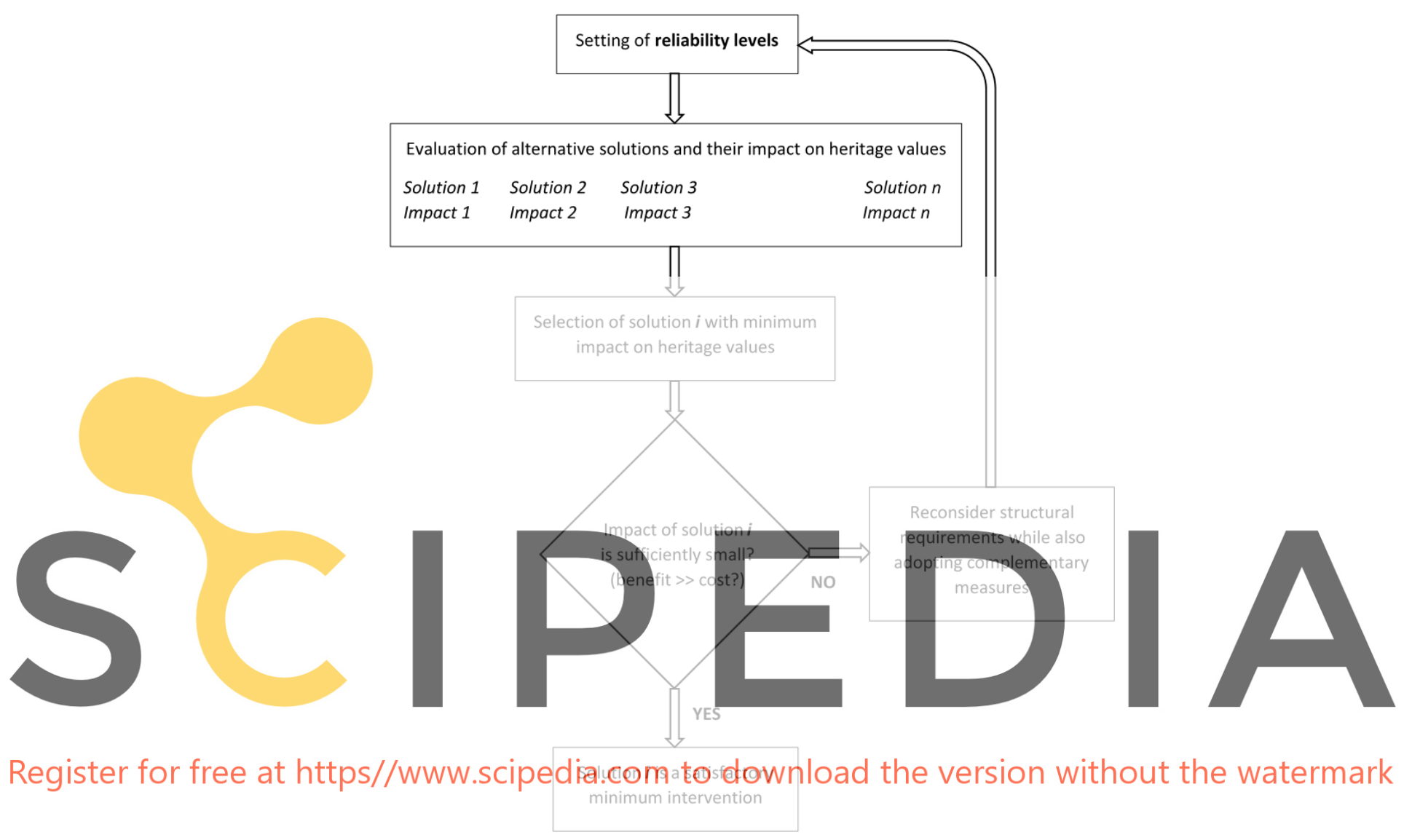

Figure 3: Engineering approach fort the selection of minimum interventions

\subsection{Incremental approach}

A different strategy towards minimum intervention can result from applying an incremental (or step -by-step) approach beginning with a minimum level of intervention and then followed by possible subsequent supplementary or corrective measures. Observation and monitoring are used to assess the efficiency of each subsequent measures and to evaluate the need for further operation. It must be noted that this approach is applicable to problems involving static loads and cannot be applied to seismic strengthening or interventions against other natural hazards.

\subsection{Impact of interventions on heritage values}

The engineering approach presented in section 7.3 is based on the evaluation of the impact that possible interventions have on the structure's heritage values. This evaluation can be based on a qualitative classification allowing to categorize the interventions according to their 
foreseeable impact. A quantitative evaluation of the impact, while desirable for the sake of objectivity and accuracy, may be difficult to perform due to the multidimensional character of the heritage values and the multiplicity of impacts that interventions may have on them.

As a first approach the guidelines propose a classification in three categories corresponding to low, moderate or high impact ones. It is also acknowledged that interventions may have, in some cases, a positive impact and may even contribute to the cultural value to the historical structure.

The first category includes interventions that have a positive or neutral impact or, if negative, a very low impact on the heritage values. The guidelines provide different criteria for the identification and classification of this type of interventions. In short, they include maintenance, stabilization, repair or strengthening operations that satisfy certain requirements regarding the criteria introduced in section 7.1. Stabilization operations should be fully non-invasive and removable. Repair operations should be based on proven compatible and durable materials (as for instance grouting and injection with adequate mortars or grouts). Strengthening interventions should satisfy several requirements, including the use of non-invasive and removable reinforcing devices (such as tie rods in masonry arches and buildings).

The second category refers to interventions with moderate impact. Included in this category are those based on light, scarcely invasive and mostly removable devices. The damage caused by the implementation or removal of the intervention should be limited and repairable by means of appropriate conventional techniques.

Other interventions that do not satisfy the aforementioned conditions may have a very high impact on the structure's cultural value and are therefore included in the third category. They include, for instance, interventions whose implementation causes significant damage (or even moderate damage if not repairable by means of appropriate conventional techniques), interventions causing a substantial alteration of the structural scheme and resisting mechanisms, or invasive and/or non-removable interventions based on not sufficiently durable or compatible materials.

\subsection{Quality assurance}

It is recognized that interventions in ancient buildings are generally more complex than in standard construction, and require all involved agents to implement appropriate methods and technologies. The success of interventions is seriously compromised if they are not entrusted to agents with the necessary qualifications in three main areas: (i) studies and design, (ii) inspection and testing, and (iii) execution. The members of the multidisciplinary team should possess the necessary qualifications on training and experience in their areas of expertise.

\section{EXPLANATORY REPORT}

The guidelines propose that any study and intervention on a historical structure should be completed with an Explanatory Report including a summary with all the findings of the multidisciplinary team. The purpose of the explanatory report is to explain those aspects that cannot be reduced to formal calculations. The report must explicitly present and discuss the reliability of the data, any uncertainties and the hypotheses considered at each stage. Among other aspects, it should include the following items: 
- The conclusions of diagnosis on the causes of decay and damage, along with an evaluation of the monument's conservation condition.

- A justified statement on the adopted reliability levels (structural requirements). If these requirements are reduced with respect to the application of current design codes in order to limit excessive intervention, it will be necessary to clearly justify this decision and to explain how adequate safety is provided by alternative means.

- The conclusions on the structural verification, along with the identification of the intervention needs.

- The criteria and procedure considered in the evaluation of the alternative solutions and the selection of the optimal one.

- A detailed description of the selected intervention.

\section{CONCLUSIONS}

Based in the ICOMOS/ISCARSAH Principles for the Analysis, Restoration and Structural Conservation of Architectural Heritage, the guidelines of the ISCASAH committee are intended to provide insight on the different stages that concern the study and conservation of historical structures, including the investigation and documentation works, the structural verification and the design of intervention. Across of these stages, the guidelines propose the application of a broad and flexible approach strongly based on the scientific method. The proposed approach includes different activities and approaches (such as the historical research, inspection, comparative analysis, monitoring and structural analysis) providing both qualitative and quantitative evidence. The outcome of all the different activities has to be integrated in a consistent way. Possible contradictions (for instance, between historical research and structural analysis) should motivate the reconsideration of the conclusions attained and a more accurate application of approaches and methods.

The proposed engineering approach for the identification of adequate minimum interventions is based on the evaluation (even if only qualitative) of the impact that the envisaged operations (of maintenance, stabilization, repair or strengthening) may have on the heritage values. Criteria are provided to categorize the interventions according to their impact. A distinction is proposed among null to low, moderate and high impact interventions. It is also acknowledged that interventions may have a positive impact on the heritage values.

\section{REFERENCES}

[1] Principles for the analysis, conservation and structural restoration of architectural heritage. (2003). ICOMOS / ISCARSAH Committee. Ratified by the ICOMOS 14th General Assembly, in Victoria Falls, Zimbabwe, October 2003

[2] ISO 13822:2010 Bases for Design of Structures - Assessment of Existing Structures. Technical Committee ISO/TC 98, Subcommittee SC2 - Reliability of Structures.

[3] International Charter for the Conservation and Restoration of Monuments and Sites (The Venice Charter, 1064). 2nd. International Congress of Architects and Technicians of Historic Monuments, Venice, 1964. Adopted by ICOMOS in 1065.

[4] The Nara Document on Authenticity (1994) / ICOMOS. Ratified at Nara Conference on Authenticity in Relation to the World Heritage Convention, held at Nara, Japan, from 1-6 November 1994 